\title{
Awareness and uptake of recommended vaccines among immunosuppressed patients
}

\author{
${ }^{1} \mathrm{~J}$ Savage, ${ }^{2} \mathrm{~F}$ Muller, ${ }^{3} \mathrm{AD}$ Ormerod \\ ${ }^{1,3}$ Division of Applied Medicine, University of Aberdeen, UK; ' ${ }^{2}$ epartment of Dermatology, Aberdeen Royal Infirmary, Aberdeen, UK
}

\begin{abstract}
The Green Book recommended in 2009 that immunosuppressed patients should be receiving the yearly seasonal flu vaccine, the pandemic swine influenza A HINI/09 vaccine and should have had the single pneumococcal vaccination. A retrospective audit in 2010 involving 60 immunosuppressed patients revealed that $83.3 \%$ of participants were aware of their entitlement to the vaccines. The majority were informed by their GP practice rather than the prescribing specialist. In $2009,70 \%$ of participants received the seasonal flu vaccine, $40 \%$ received the $\mathrm{HINI}$ vaccine and $21.6 \%$ had received the pneumococcal vaccine. Reasons given for not receiving the recommended vaccines were lack of awareness, reported by $37.5 \%$, followed by worries regarding side-effects reported by $25 \%$. The data suggest that uptake rates, particularly for pneumococcal vaccination, could be improved with targeted information and promotion at the point of commencing immunosuppressants and approaching the influenza season. Prescribing physicians should take a more active role in routinely promoting and planning vaccination for at-risk groups and should provide information on how to receive the recommended vaccines and their side-effect profiles.
\end{abstract}

KEYWORDS Pandemic influenza, A HINI/09, seasonal influenza, pneumococcal vaccine, immunosuppressant

DECLARATION OF INTERESTS Dr Ormerod has received travel and lecture fees from Abbott, Pfizer, Jansen-Cilag and departmental support for randomised control trials in biologics from the above, as well as MSD. Dr Muller has received travel and accommodation fees from Basilea and Cephalon.

\section{INTRODUCTION}

Immunosuppressants and biologics are prescribed in a wide range of patients, including those with chronic inflammatory diseases such as Crohn's and rheumatoid arthritis, patients with severe psoriasis and patients who have received an organ or bone marrow transplant. Physicians should be familiar with the Department of Health publication Immunisation Against Infectious Disease, more commonly known as The Green Book, which provides the standard regarding what vaccinations patients on biologics and immunosuppressants should be receiving to prevent avoidable infections. All live vaccines are contraindicated until a minimum of six months after immunosuppressive drugs have been withdrawn.' However, there are three vaccines which are recommended for all patients on immunosuppressant medications: the one-off pneumococcal vaccine, the yearly seasonal flu vaccine and, since the World Health Organisation (WHO) announced the HINI pandemic in June 2009, the recommendations advised that these patients should also be offered the pandemic (swine) influenza $A$ HINI/09 vaccine. ${ }^{2}$ In 2010 the influenza $A$ $\mathrm{HINI} / 09$ was included in a trivalent influenza vaccine including the synomonous A/California/7/2009 (HINI) together with A/Perth/16/2009 (H3N2) and B/Brisbane/ $60 / 2008$ and is modified according to viruses circulating and antigenic drift each year. Vaccination rates are likely
Correspondence to A Ormerod, Division of Applied Medicine, University of Aberdeen, Polwarth Building, Foresterhill, Aberdeen AB25 2ZN, UK

tel. +44 (0) I 224553955 e-mail a.d.ormerod@abdn.ac.uk to have been modified by the perceived threat of a major epidemic in 2009, 40 years since the previous Hong Kong influenza pandemic. However, the predicted consequences of the 2009 pandemic were not realised and this could have led to complacency in 2010. Recent data from the Health Protection Agency confirms that 2010/II had a higher incidence of influenza with influenza A HINI/09 still in circulation, affecting more young people, leading to increased intensive care bed occupancy and more deaths than the previous year. The data confirm the efficacy of annual vaccination and that immunosupression is the highest risk factor for mortality (age-adjusted risk ratio $47.395 \% \mathrm{Cl} 35.3-63.1$ ). ${ }^{3}$

All of the vaccines recommended for the immunosupressed are inactivated. Absolute contraindications are confirmed anaphylaxis to a specific vaccine or its components. In the case of the seasonal influenza vaccine, an anaphylactic reaction to eggs is also an absolute contraindication. However, individuals can still be vaccinated against $A$ / California/07/2009 (HINI)v using celvapan. The pneumococcal vaccine protects against Streptococcus pneumoniae, which can cause pneumonia, bacteraemia or meningitis in susceptible individuals ${ }^{2}$ and is ideally given before therapy with immunosuppressants is commenced.'

Due to the morbidity, mortality and pressure on healthcare represented yearly by influenza, there have 


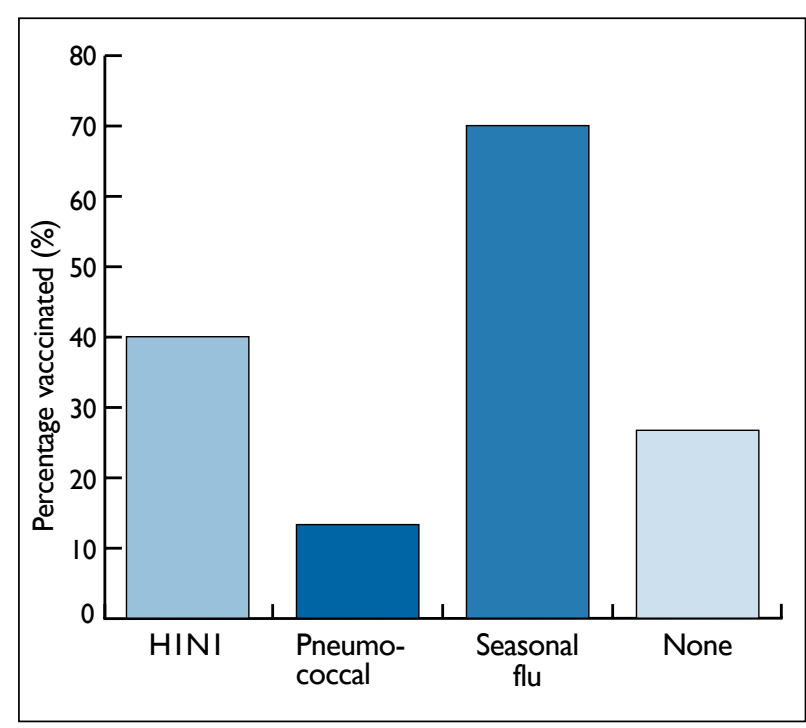

FIGURE I Percentage uptake of pandemic influenza A $\mathrm{HINI} / 09$, pneumococcal and seasonal flu vaccines.

been a number of studies into its uptake by different groups. ${ }^{4-10}$ The seasonal influenza vaccine is also recommended for patients with chronic medical conditions, pregnant women, the elderly and healthcare professionals. There is little information available on vaccine uptake by immuno-suppressed patients. ${ }^{5}$ However, despite evidence of increased uptake of influenza vaccine by at-risk groups in the UK, higher than in most European Union countries ${ }^{4}$, some data suggest that patients on immunosuppressants have a lower uptake $(44.9 \%$ I $6-65 \mathrm{yrs}){ }^{5}$

\section{METHODS}

The study population consisted of attendees at outpatient dermatology clinics in Aberdeen Royal Infirmary (ARI). A questionnaire was distributed to dermatologists with clinics at ARI between August 30 and October 242010 . Practitioners were asked to give it to patients currently managed on immunosuppressants and biologics. Further participants were recruited through the mail. Seventy questionnaires were posted along with stamped and pre-addressed return envelopes and a cover letter from the patient's own dermatologist requesting their participation. The questionnaire requested details on the participant's awareness of entitlement to the HINI, pneumococcal and seasonal flu vaccines, how they were made aware of these vaccines and whether they received each of these vaccines between October 2009 and 20I0. It also asked about reasons for non-uptake, intentions to receive the recommended vaccines in the future and requested participants gender and age. All data collected were anonymous and patients were only approached by their treating dermatologist. Information was entered into an Excel spreadsheet, constructed with input from NHS Grampian's Department for Clinical Effectiveness, for data analysis.

\section{RESULTS}

Eighty-seven questionnaires were distributed, 63 were completed (of which three were excluded due to incomplete sections) giving a $69 \%$ response rate. The sample population consisted of 36 men and 24 women aged over 16 . In total, 50 out of 60 (83.3\%) of participants were aware that they should receive the recommended vaccines. Of these, the majority $(68 \%)$ were alerted by their GP practice and a further $24 \%$ by the prescribing dermatologist. In total, 42 of the 60 participants (70\%) received the seasonal flu vaccine in the 2009-2010 round of vaccinations and 24 out of 60 (40\%) participants received the pandemic influenza $A \mathrm{HINI} / 09$ vaccine in the 2009-2010 round of vaccinations. Thirteen out of 60 participants $(21.6 \%)$ had received the pneumococcal vaccine in the previous year, while $26.7 \%$ did not receive any of the vaccines (Figure I).

Uptake was similar in males and females: $70.8 \%$ of the women who took part received the seasonal flu vaccine compared to $69.4 \%$ of men; $41.7 \%(90 \% \mathrm{Cl} 25.1-58.2)$ of the women received the HINI vaccine compared to $58.3 \%$ ( $90 \% \mathrm{Cl} 44.8-7 \mathrm{I} .8$ ) of men; I $2.5 \%$ of the women received the pneumococcal vaccine compared to $13.8 \%$ of men; $25 \%$ of the women received none of the available vaccines compared to $27.7 \%$ of the men.

Younger patients were more likely to receive none of the recommended vaccines. Patients in the older age groups received the seasonal flu vaccination more often. For every vaccine, the highest percentage uptake was in the over 65 age group with the highest pandemic influenza A HINI/09 vaccine uptake in the 66-75 year olds and the highest seasonal flu and pneumococcal vaccine uptake levels in the over 75s (Figure 2).

Of those who were not vaccinated, $50 \%$ attributed this to being unaware that they were recommended, $25 \%$ to worries about side-effects and $12.5 \%$ to being unsure of how to arrange to receive the vaccines. A total of $6.3 \%$ were unwell at the time of their vaccine appointment and $6.3 \%$ had contraindications. Forty-one out of 60 (68.3\%) participants planned to have one or more of the recommended vaccines in the next round of vaccinations. A further $10 \%$ did not plan to have any of the vaccinations in the next round and $21.7 \%$ were undecided.

The immunosuppressive therapy of the participants were as follows: nine were on etanercept, 13 were on adalimumab, six were on infliximab, two were on ustekinumab, six were on azathioprine, nine were on ciclosporin and 15 were on methotrexate.

\section{CONCLUSION}

The WHO target for uptake of the influenza vaccine by 2010 is $75 \%$, and this audit showed an uptake just below 


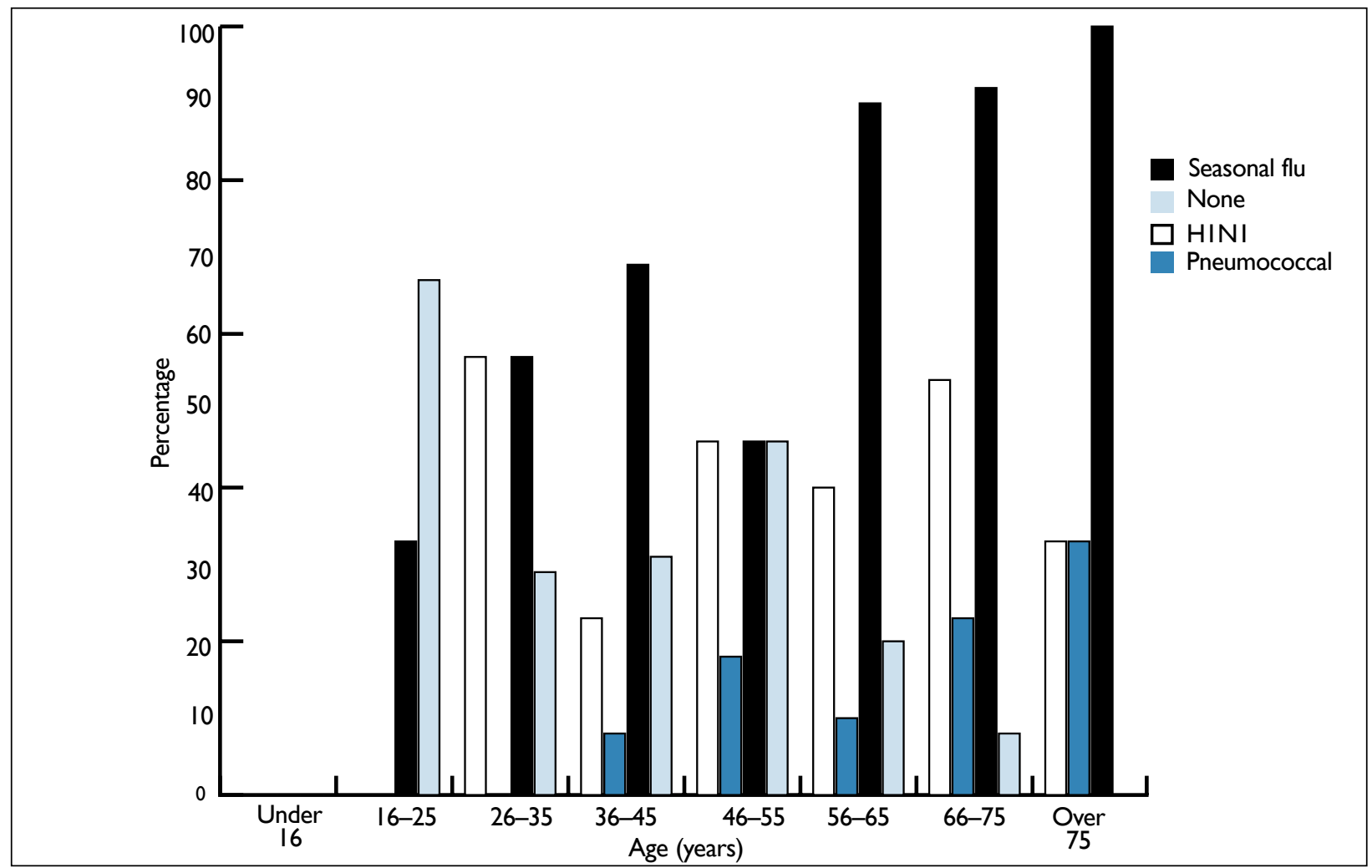

FIGURE 2 Percentage uptake of recommended vaccines by age group.

this. The uptake of $70 \%$ recorded is slightly lower than the UK-wide uptake $(74 \%)$ reported in a 2010 review of seasonal flu vaccine coverage levels. ${ }^{4}$ In 2010/II, uptake in patients aged 65 years and over increased from $2009 / 10$ to $2010 / 11$ in Scotland (from $73.6 \%$ to $75.4 \%$ ) and Wales (from $63.5 \%$ to $65.8 \%$ ). ${ }^{3}$ With the introduction of the trivalent seasonal flu vaccine, the uptake rate of pandemic influenza $\mathrm{A} \mathrm{HINI/09}$ has reverted to that for seasonal flu. The uptake by immunosuppressed patients is given in a report on seasonal influenza coverage with data from 8,293 GP practices in 2009. It quoted an uptake rate of $44.9 \%$, lower than for any other at-risk group ${ }^{5}$ and similar to that reported in England in 2010/II (in the under 65s at $50.4 \%$ of at-risk groups).These figures are significantly lower than we found in this audit and suggest that we are achieving a better uptake. However, this could be further improved. As only 10\% of the group have decided against vaccination, a potential $90 \%$ vaccination rate should be achievable in this select group.

Respondents reported lower levels of uptake of pandemic influenza $\mathrm{A} \mathrm{HINI/09}$ and pneumococcal vaccines compared to the seasonal flu or influenza vaccine. Lower levels of uptake of the pandemic influenza A HINI/09 vaccine in 2009/10 were probably because this was new, leading to increased concerns regarding safety. A study of Scotland's vaccination programme in $2009 / 10$ found a $37.5 \%$ uptake of pandemic influenza A HINI/09 vaccine similar to the findings in this audit. ${ }^{10}$ Now that this is combined in a trivalent vaccine, the option not to take this part of the vaccine is no longer available, which should encourage uptake. There was a clear trend showing an increase in uptake of the seasonal flu vaccine with increasing age, concurring with other studies. ${ }^{6,11}$ This is likely to be because the seasonal flu vaccine is recommended to over $65 \mathrm{~s}$ regardless of medications or medical conditions, as part of a national programme.

A retrospective Irish telephone survey recorded pneumococcal vaccine uptake at II\% for at-risk groups under age 65 , again similar to our findings. ${ }^{7}$ A postal survey into uptake by the over $65 \mathrm{~s}$ in nursing homes showed a $34 \%$ uptake level for the pneumococcal vaccine." The low uptake of pneumococcal vaccine could also be accounted for by the greater publicity and national programmes about the seasonal flu vaccine.

Lack of uptake was most commonly attributed to lack of awareness. To achieve better uptake we recommend that when the physician decides to use immunosupressives, the patient should have vaccines routinely recommended in a letter to the general practitioner. They should also be informed about the side-effects, benefits and when and how to receive the vaccines. General practitioners should add the names of such patients to their vaccine list. At review, the physician should take every opportunity to remind patients when they are due and check that they have received appropriate vaccines. 
We need to actively promote the uptake of seasonal influenza vaccines in the younger patients who appear to be more at risk of death from the newer strains of influenza, and the use of pneumococcal vaccination in all patients receiving immunosupressants. A similar audit should be repeated when measures are in place.

\section{REFERENCES}

I Smith $\mathrm{CH}$, Anstey AV, Barker JN et al. British Association of Dermatologists' guidelines for biologic interventions for psoriasis 2009. BrJDermato/2009; |61:987-1019.doi:I0.I I I I/j. I365-2133.2009.09505.x

2 Department of Health. Immunisation against infectious disease [Internet]. Department of Health; 2006. [cited 20II March 3I] Available from: http://www.dh.gov.uk/en/Publichealth/Immunisation/ Greenbook/index.htm

3 Health Protection Agency. Surveillance of influenza and other respiratory viruses in the UK: 2010-201I report [Internet]. Health Protection Agency; 20II. [cited 20II May 26]. Available from: http://www.hpa.org.uk/web/HPAweb\&HPAwebStandard/ HPAweb_C/I2966874I2376

4 Monto AS. Seasonal influenza and vaccination coverage. Vaccine 2010; 28:D33-44. doi:I0.1016/j.vaccine.2010.08.027

5 Gates P, Noakes K, Begum F et al. Collection of routine national seasonal influenza vaccine coverage data from GP practices in England using a web-based collection system. Vaccine 2009; 27 : 6669-77. doi:10.1016/j.vaccine.2009.08.094

6 Joseph C, Goddard N, Gelb D. Influenza vaccine uptake and distribution in England and Wales using data from the General Practice Research Database, 1989/90-2003/04. J Public Health 2005; 27:37I-77. doi:10.1093/pubmed/fdi054
The small numbers involved in this study and its retrospective nature limits the ability to base any generalisations upon the results presented. However, the importance of vaccination applies across many hospital specialties and not enough attention has been given to this in the literature.

7 Mereckiene J, O'Donnell J, Collins $C$ et al. Risk groups and uptake of influenza and pneumococcal vaccine in Ireland. Euro Surveill 2007; I2:EI3-14.

8 Maurer J, Uscher-Pines L, Harris KM. Perceived seriousness of seasonal and $\mathrm{A}$ [HINI] influenzas, attitudes towards vaccination, and vaccine uptake among US adults: does the source of information matter? Prev Med 2010; 5I:185-87. doi:10.1016/j. ypmed.2010.05.008

9 Maurer J, Harris KM, Parker A et al. Does receipt of seasonal influenza vaccine predict intention to receive novel $\mathrm{HINI}$ vaccine: evidence from a nationally representative survey of US adults. Vaccine 2009; 27:5732-34. doi:10.1016/j.vaccine.2009.07.080

10 Simpson C, Ritchie L, Robertson C et al. Vaccine effectiveness in pandemic influenza - primary care reporting [VIPER]: an observational study to assess the effectiveness of the pandemic influenza A [HINI]v vaccine. Health Technol Assess 20I0; I4:3I3-46.

II Shroufi A, Copping J, Roberto Vivancos R et al. Influenza and pneumococcal vaccine uptake among nursing home residents in Nottingham, England: a postal questionnaire survey. BMC Geriatr 2008; 8: I I. doi: I0.II86/I47II-23|8-8-II 